\title{
Effect of ablation time and laser fluence on the optical properties of copper nano colloids prepared by laser ablation technique
}

\author{
H. S. Desarkar • P. Kumbhakar • A. K. Mitra
}

Received: 29 November 2011 / Accepted: 23 March 2012/Published online: 5 April 2012

(C) The Author(s) 2012. This article is published with open access at Springerlink.com

\begin{abstract}
Copper $(\mathrm{Cu})$ nanoparticles of average sizes (radius in $\mathrm{nm}$ ) varying between 1.7 and $6 \mathrm{~nm}$ have been prepared by 1,064 nm Nd:YAG laser ablation of solid copper target in water medium. The nanostructures of the samples have been characterized using high-resolution transmission electron microscopes (HRTEM). The UVvisible absorption spectra obtained with a UV-visible spectrophotometer show sharp absorptions in the ultraviolet region and visible region due to the interband transition and surface plasmon resonance (SPR) oscillations in $\mathrm{Cu}$ nanoparticles, respectively. The increase in the linewidth of the SPR absorption peaks with the reduction in particle sizes are observed due to the intrinsic size effects. The behaviour of the UV-visible spectra associated with the $\mathrm{Cu}$ nanoparticles is studied as a function of laser fluence and laser ablation time.
\end{abstract}

Keywords $\mathrm{Cu}$ nanoparticles · Laser ablation . Surface plasma resonance $\cdot$ Interband transition

\section{Introduction}

In the past two decades metal nanoparticles have attracted much attention from the researchers because of their sizedependent electronic, catalytic, magnetic and optical

A. K. Mitra: Retired.

H. S. Desarkar · P. Kumbhakar $(\square)$ · A. K. Mitra Nanoscience Laboratory, Department of Physics, National Institute of Technology, Durgapur 713209, India e-mail: nitdgpkumbhakar@yahoo.com;

pathik.kumbhakar@phy.nitdgp.ac.in properties that arise due to interaction between metallic nanoparticles and electromagnetic radiation (Link and ElSayed 1998; Takami et al. 1999; Curtis et al. 1988; Yeh et al. 1999; Singh et al. 2010; Lee and El- Sayed 2006; Lee et al. 2006). A number of synthesis techniques for metallic colloids have been reported, such as wet chemical (Ahmadi et al. 1996), photochemical (Huang et al. 1996), electro chemical (Khaydarov et al. 2009) and radiolysis reduction (Chen et al. 2007), etc. Laser ablation of solids in a liquid (LAL) is a new and highly promising technique for preparing nanocolloids. It is of particular interest due to its high non-equilibrium processing which allows synthesis of nano-structured material. A series of mechanisms including generation, transformation and condensation of plasma plume produced laser ablation of solid in liquid environment in the condition of liquid confinement. This method allows us to prepare nanocolloids with ease and eliminates the problem of contamination by reducing agent, but the size distribution tends to be broadened because the coagulation processes of the atoms can hardly be controlled. This process can be used for metals, semiconductor, insulators and the conditions during ablation can be easily controlled. Among the variety of metallic nanoparticles noble metal group gold $(\mathrm{Au})$ and silver $(\mathrm{Ag})$ are extensively studied as they interact strongly with visible light through resonant excitations of the collective oscillation of the conduction electrons within the particles. Recently attention has been focused on $\mathrm{Cu}$ nanocolloids due to their catalytic (Mafune et al. 2000) and electrolytic (Kazakevich et al. 2004) properties. Formation of nanocolloids under pulsed Nd:YAG laser ablation of metallic plates in liquid has been reported, previously (Link et al. 2000; Dolgaev et al. 2002; Nedersen et al. 1993). Recently Jeon et al. (1998) have reported the effect of different laser wavelength $(1,064$ and $532 \mathrm{~nm})$ and for a particular wavelength 
$(1,064 \mathrm{~nm})$ the effect of focusing on ablation efficiency. Haider et al. (2011) have reported the effect of laser energy on the ablation efficiency of the prepared gold nanocolloids. Very recently, Mafune et al. (2002) has reported the formation of $\mathrm{Ag}$ nanocolloids in aquash solution of sodium dodecyl sulphate (SDS) and examined the influence of laser energy, number of laser pulses and concentration of SDS on ablation efficiency. Prochazska et al. (1997) have reported the wavelength dependence on ablation efficiency of various metals.

In this study we demonstrate a method to generate $\mathrm{Cu}$ nanoparticles of average sizes (radius) varying between 1.7 and $6 \mathrm{~nm}$ by $1,064 \mathrm{~nm} \mathrm{Nd}$ :YAG laser ablation and observed the appearance of strong and sharp interband absorption in the ultraviolet region at $\sim 251 \mathrm{~nm}$ $(\sim 4.94 \mathrm{eV})$ as well as SPR absorptions in the visible region (593-607 $\mathrm{nm}$ ). It is found from the measured UVvisible absorption characteristics of all the samples that the SPR peak positions and thus the particle sizes of the prepared nanoparticles are dependent on the laser ablation time (LAT) and laser fluence (LF). In order to understand the effect of laser parameters on mean nanocolloids' size a series of laser ablation experiment is performed. The variations in size of the prepared $\mathrm{Cu}$ nanoparticles have been obtained by varying the LAT from 10 to $50 \mathrm{~min}$, keeping the LF constant at $151 \mathrm{~J} / \mathrm{cm}^{2}$. The observed results have been analysed using the frame of Mie theory and the value of scattering parameter and bulk copper damping frequency have been extracted. It is found that SPR linewidth increases with the decrease in particle size due to intrinsic size effects. We have studied the effect of the LF keeping the ablation time duration fixed at $20 \mathrm{~min}$. Despite the complex mechanism of laser-matter interaction, laser ablation in liquid technique provides a simple, flexible and less expensive way for formation of contamination free nanoparticles.

\section{Experimental details}

The experimental set up used for preparation of $\mathrm{Cu}$ nanocolloids is shown in the Fig. 1a. The solid $\mathrm{Cu}$ target used is a Cu plate of $\sim 10 \mathrm{~mm}$ thickness and of purity of $99.99 \%$ which is kept in a petri dish containing $10 \mathrm{ml}$ of liquid. The laser radiation at $1,064 \mathrm{~nm}$ wavelength coming from a Q-switched Nd:YAG laser is focused using a lens of focal length $30 \mathrm{~cm}$. The formations of nanoparticles along-with its size variation with the time of ablation are visually noted with the variation of colour of the prepared colloids. The laser source is operated at a repetition rate of $10 \mathrm{~Hz}$ and ablation time was varied between 10 and $50 \mathrm{~min}$. As the laser radiation is incident on the target for long exposure time, crater is formed on the target. As a result the value of the laser fluence reaching the target will change and which may reduce the formation efficiency of the nanoparticles (Haider et al. 2011; Mafune et al. 2002). In order to avoid this, petri dish containing the target is translated. The nanostructures of the samples are obtained using a transmission electron microscope (TEM, FIB-20). The micrograph is analysed by image processing software to determine the mean particle size. The size distribution is obtained by counting at least 250 particles in TEM image. It should be noted that both the size and the size distribution significantly depend on the laser parameters. The UVvisible absorption characteristics have been measured using a UV-visible spectrophotometer (Hitachi, U 3010) and for these measurements, nanocolloids are kept in a quartz cuvette of path length $1 \mathrm{~cm}$.

\section{Results and discussions}

The energy-dispersive X-ray analysis (EDXA) spectrum of a representative sample, prepared by $30 \mathrm{~min}$ of laser
Fig. 1 a The schematic of the experimental set up for the preparation of $\mathrm{Cu}$ nanocolloids by laser ablation. b Shows the EDXA spectrum of a sample S3
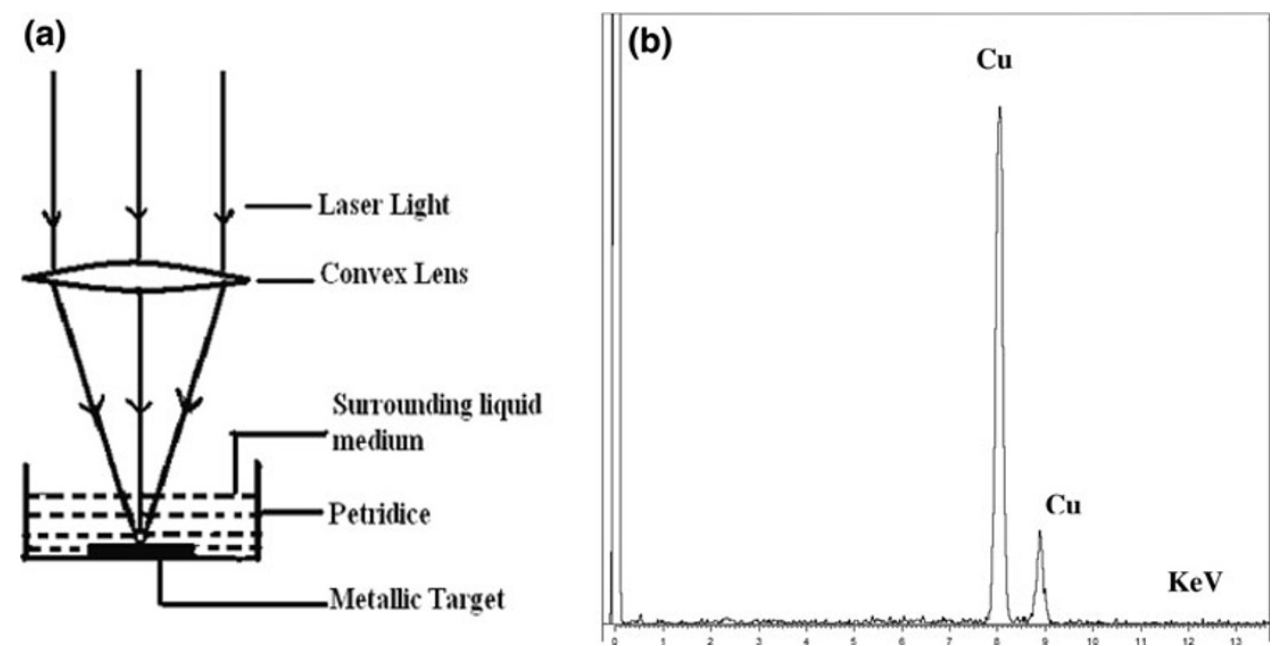
ablation, is shown in Fig. 1b. The EDXA spectrum shows the presence of $\mathrm{Cu}$ as principal element. Figures $2 \mathrm{a}, \mathrm{b}, \mathrm{c}, \mathrm{d}$ and e show the particle size distributions (with the corresponding TEM micrographs in the inset) of the samples S1, S2, S3, S4 and S5, respectively, prepared with different LAT 10, 20, 30, 40 and $50 \mathrm{~min}$ and with fixed LF of 151 $\mathrm{J} / \mathrm{cm}^{2}$. The mean size and the size distribution of the particles are obtained by counting more than 250 particles. Histogram for each samples have been plotted on taking the maximum lateral width of the particle. Histograms of the size distribution at different LAT are fitted to a lognormal size distribution in our statistics. Most of the particles have the diameter about $3.4-12 \mathrm{~nm}$ with an asymmetric distribution of sizes. It is found from TEM micrograph that there are some occasional particles as large as $30 \mathrm{~nm}$. Some of the nanoparticles are loosely agglomerated, and some present chain of particles.

Structural information from an assembly of $\mathrm{Cu}$ nanoparticles is obtained using selected area electron diffraction (SAED) pattern. The pattern consists of five resolved concentric rings. The inset of Fig. 2c shows the SAED pattern of sample S3. The calculated values of the lattice spacing obtained from the five different rings in the SAED pattern are compared with the standard lattice spacing of $\mathrm{Cu}$ (JCPDF 04-784) and $\mathrm{Cu}_{2} \mathrm{O}$ (JCPDS 05-667) along with their respective $(h k l)$ indices and the results are summarized in Table 1. From the Table 1 it is found that our calculated values match exactly well with those of standard value of $\mathrm{Cu}$ and $\mathrm{Cu}_{2} \mathrm{O}$ which confirms the coexistence of both $\mathrm{Cu}$ and $\mathrm{Cu}_{2} \mathrm{O}$ rings. It suggests that the surfaces of $\mathrm{Cu}$ nanoparticles are partly oxidized by water because of high reactivity of $\mathrm{Cu}$.

Figure 3 shows the UV-visible absorption characteristics of all as-prepared samples. In the Fig. 3, dark yellow, red, black, blue and magenta colours correspond to S1, S2, S3, S4 and S5, respectively. From Fig. 3 it is seen that there are two absorption peaks appearing in all the samples, one in the visible region due to the well-known SPR absorptions, which is the characteristics absorption of metals and another in the UV region due to interband transitions. The appearance of sharp and strong interband transition in the UV region is an important observation in this work.

From the inset of Fig. 3 it is seen that the maximum value of absorbance of SPR and UV peaks vary with LAT. It is also found from the inset of Fig. 3 that UV absorption peak intensity increases from S1 to S3 and after that it decreases for S4 and then again increases for S5. It is also observed that SPR absorption peak intensity increases slowly from S1 to $\mathrm{S} 4$ and then it almost becomes constant. These results indicate that as we have increased the ablation time, the number of nanoparticles produced increases initially and then it becomes almost constant with ablation time. Actually, with increase in laser ablation time, more number of nanoparticles are produced and these nanoparticles accumulate near the laser spot; when the number of produced nanoparticles becomes sufficiently large, they shield the incident laser radiation. Hence the value of the incident laser intensity reaching the solid target is decreased, which causes no further increase in the number of nanoparticles and so the absorbance becomes constant.

Figure 4a shows the variations of the SPR wavelengths and size of the particles (diameter in $\mathrm{nm}$ ) with the time duration of laser ablation for all the samples. It is observed that the SPR peak position shows a gradual blue shift with ablation time and the particle size (diameter in $\mathrm{nm}$ ) as obtained from TEM measurements decreases with LAT. The blue shift of SPR peak position due to the reduction of the size of the prepared nanoparticles agrees with the classical plasmon resonance absorption theory of metallic colloidal particles (Link and El-Sayed 1999; Kreibig and Genzel 1985). The interband transition in UV region has been reported in chemically prepared $\mathrm{Au}$ and $\mathrm{Ag}$ nanoparticles and in $\mathrm{Au}$ nanoparticles deposited in a quartz substrate by RF sputtering (Balamurugan and Maruyama 2005; Frederix et al. 2003; Alvarez et al. 1997). The surfactants and capping molecules have also been observed to influence the intensity and width of the interband absorption peak and this phenomenon has been successfully used for biosensor applications (Pinchuk et al. 2004). Also it has been reported theoretically that there is a considerable contribution of interband transition to the absorption spectra of $\mathrm{Au}$ and $\mathrm{Ag}$ nanoparticles at UV wavelengths (Rao et al. 2002). In the present work, no surfactant or capping molecules have been used and so the intense interband absorptions are observed and their intensity also varies with the laser ablation time duration. The UV peak absorbance is found to increase from S1 to S3. Then absorbance is suddenly reduced for S4 due to agglomeration of nanoparticles caused by shielding of the laser beam. The UV absorption in the sample prepared with longer time duration is again increased due to fragmentation of the agglomerated nanoparticles. The electronic energy levels are not continuous in nanoparticles unlike in bulk materials. Rao et al. (2002) reported that due to the quantum size effects of the careers, the energy levels are discrete and this can result in a sharp and intense absorption features corresponding to interband transitions in nanoparticles.

The linewidth of the SPR peak is an important parameter to have an idea about the size distribution of the prepared nanocolloids. We have applied Lorentzian fit to calculate the Lorentzian linewidth (in meV) of SPR peak of all the samples and are shown in Fig. 4b with their corresponding particle size. From the Fig. $4 \mathrm{~b}$ it is observed that the linewidth increases with the decrease in particle size; thus the presence of intrinsic size effects is confirmed (Berciaud et al. 2005). With the Mie theory and the bulk dielectric constant only a minor dependence of the SPR 

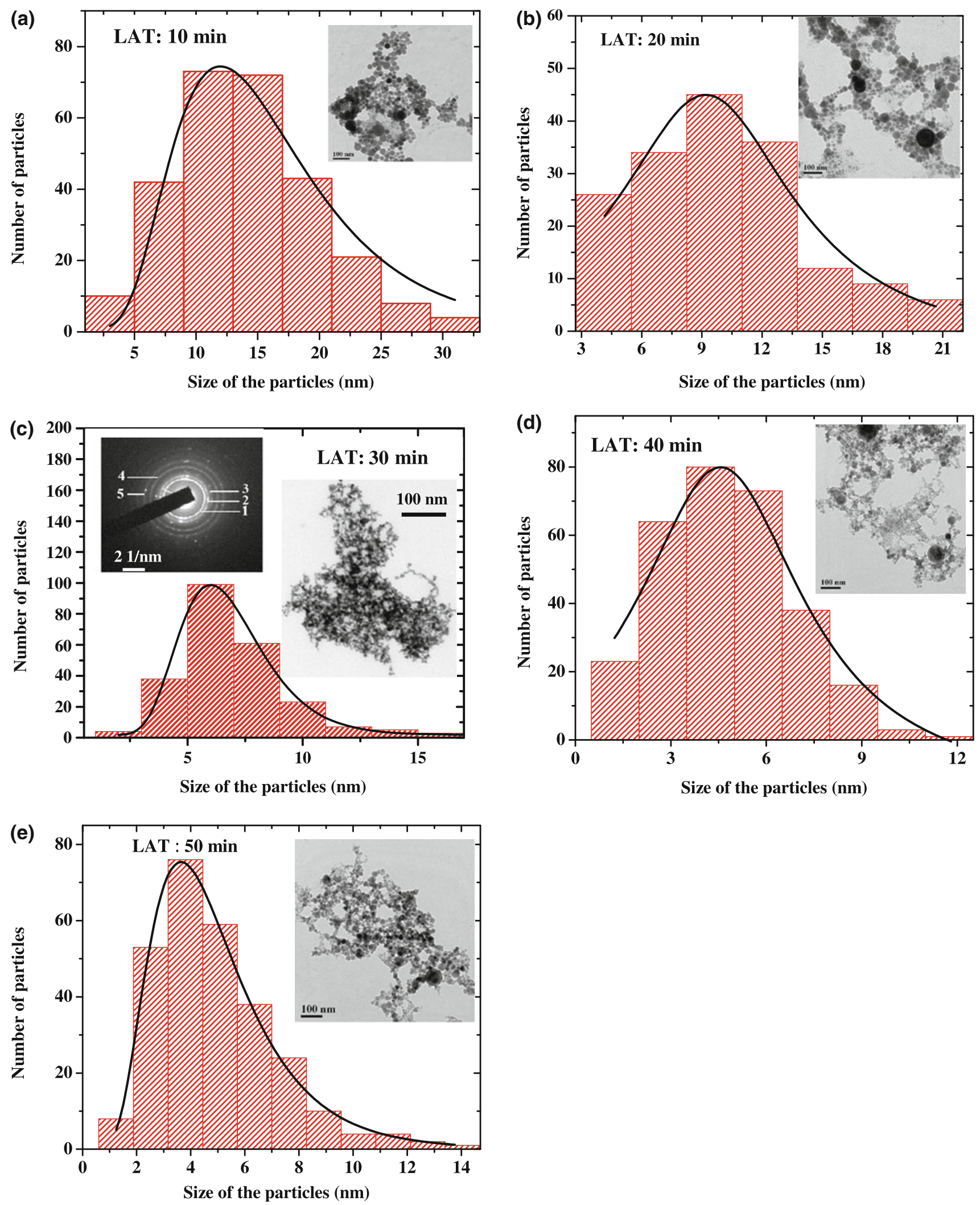

Fig. 2 Images marked as a, b, c, $\mathbf{d}$ and $\mathbf{e}$ show the particle size distributions of the samples S1, S2, S3, S4 and S5 and the corresponding TEM micrographs are shown in the insets. The SAED pattern of a representative sample S3 is shown in the inset of $\mathbf{c}$ 
Table 1 Measured lattice spacing $d(\mathrm{~nm})$ from the SAED pattern shown in Fig. 2c and standard (JCPDS 04-784) lattice spacing $\left(d_{1}\right)$ $\mathrm{nm}$ for $\mathrm{Cu}$ and $\mathrm{Cu}_{2} \mathrm{O}$ along with their respective $(h k l)$ indices

\begin{tabular}{llllll}
\hline Lattice spacing's $(\mathrm{nm})$ & \multicolumn{6}{l}{ Rings } & & & \\
\cline { 2 - 6 } & 1st & 2nd & 3rd & 4th & 5th \\
\hline$d(\mathrm{~nm})$ & 0.246 & 0.208 & 0.18 & 0.15 & 0.127 \\
$d_{1}(\mathrm{~nm})$ of $\mathrm{Cu}$ & - & 0.208 & 0.18 & - & 0.127 \\
$d_{1}(\mathrm{~nm})$ of $\mathrm{Cu}_{2} \mathrm{O}$ & 0.246 & - & - & 0.151 & - \\
$(h k l)$ & $(111)$ & $(111)$ & $(200)$ & $(200)$ & $(220)$ \\
\hline
\end{tabular}

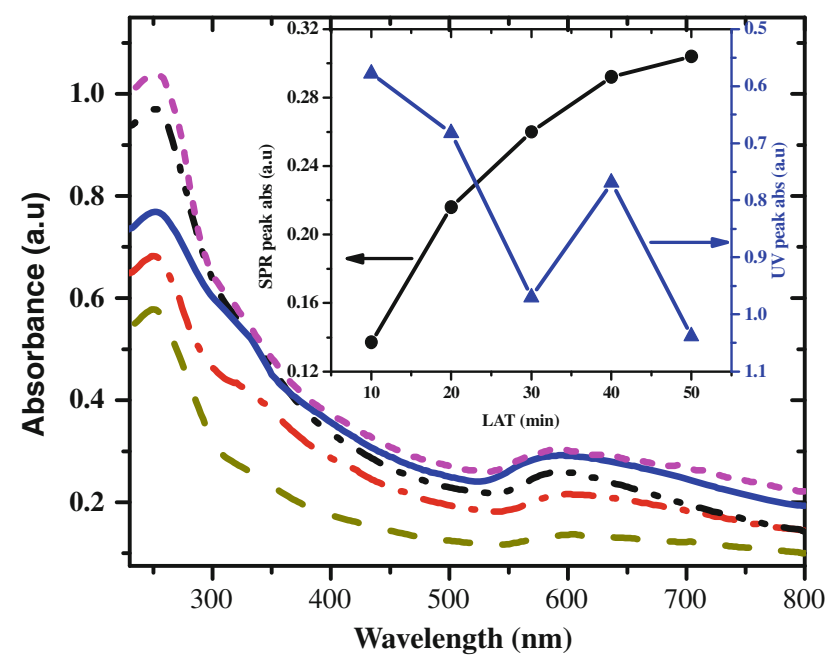

Fig. 3 Variation of absorption spectra with the laser ablation time of various $\mathrm{Cu}$ nanocolloids prepared in water medium. The colours dark yellow, red, black, blue and magenta correspond to the laser ablation time duration of 10, 20, 30, 40, and $50 \mathrm{~min}$, respectively. The inset shows the variations of the surface plasmon resonance (SPR) peak and UV peak absorptions with the laser ablation time duration

linewidth on the particle size can be explained. Therefore, to explain the experimental data of SPR linewidth we have used the modified dielectric function as given below (Kreibig and Vollmer 1995):

$\varepsilon(\omega, d)=\varepsilon_{\mathrm{ib}}+1-\frac{\omega_{\mathrm{p}}^{2}}{\omega\left(1+i \omega \gamma_{\mathrm{d}}\right)}$.

Here, the first term is the contribution to the dielectric constant due to interband transitions and assumed to be size independent and the last term is the Drude-Sommerfeld free electron term. $\omega_{\mathrm{p}}$ is the bulk plasma frequency and $\gamma_{\mathrm{d}}$ is the size dependent phenomenological damping constant as given by the following equation (Kreibig and Genzel 1985),

$\gamma_{\mathrm{d}}=\gamma_{0}+\frac{A v_{\mathrm{F}}}{r}$.

Here, $v_{\mathrm{f}}$ is the Fermi velocity and $\gamma_{0}$ is the damping constant of bulk metal, the value of $v_{\mathrm{f}}=1.57 \times 10^{6} \mathrm{~m} / \mathrm{s}$,
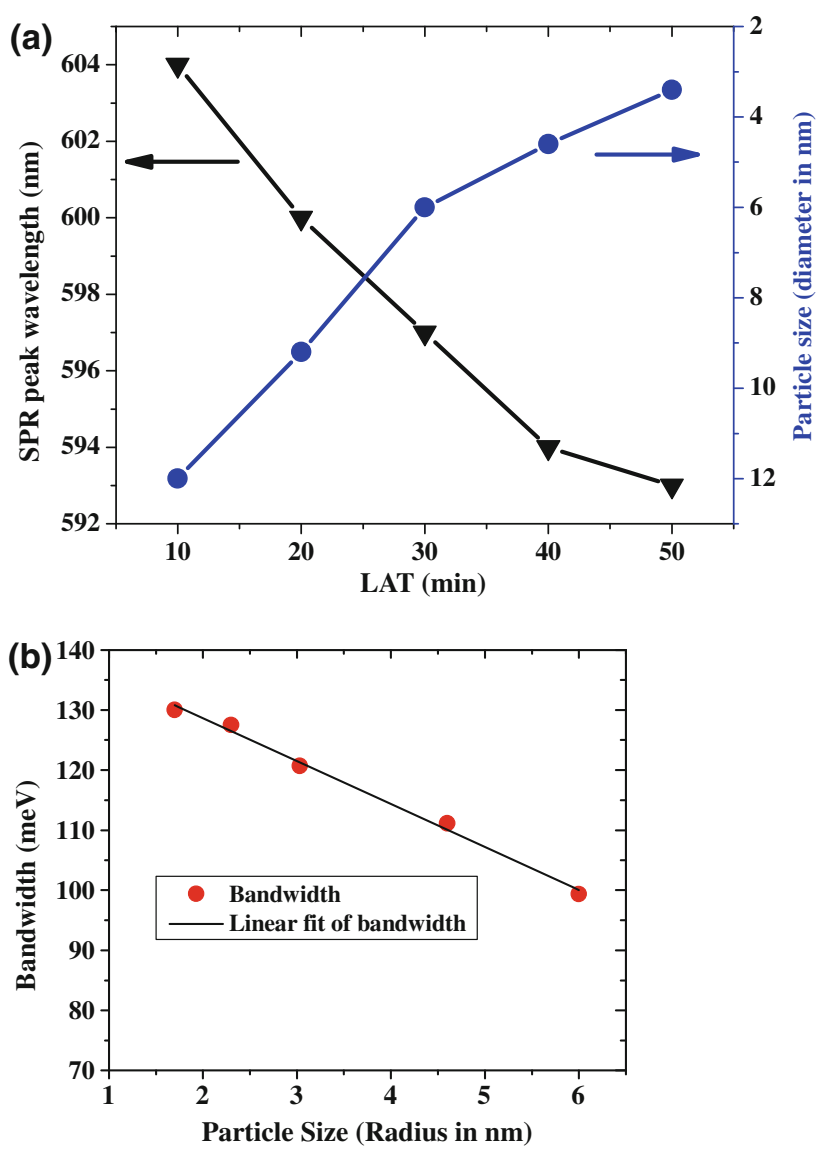

Fig. 4 Variations of SPR wavelengths and the average particle size (diameter in $\mathrm{nm}$ ) as obtained from TEM micrograph analysis with the laser ablation time duration $(\mathrm{min})$ are shown in a. Variation of the linewidth (meV) with the particle size (radius in $\mathrm{nm}$ ) is shown in $\mathbf{b}$. Symbols are experimental points and the solid line is the theoretically fitted line

and $A$ is the phenomenological parameter. The above equation described the limitations on the mean-free path of the free electrons due to the size reduction of particles. Kreibig and Genzel (1985) have shown theoretically that the values of $A$ lying between 0.1 to above 2 are justified considering several factors into the consideration. However, the circles in Fig. $4 \mathrm{~b}$ are experimental points and the solid line is the theoretically fitted one with Eq. (2). From the theoretical fittings, the obtained value of $A=0.1$ and the bulk linewidth $\gamma_{0}=89 \mathrm{meV}$ which are nearly the same as reported earlier (Yeshchenko et al. 2006).

Figure 5 shows the UV-visible absorption characteristics of all as-prepared samples prepared with different LF values of $87,121,151$ and $181 \mathrm{~J} / \mathrm{cm}^{2}$ while the LAT is kept fixed at 20 min. In Fig. 5 colors black, red, blue and dark cyan colour correspond to the LF value of $87,121,151$ and $181 \mathrm{~J} / \mathrm{cm}^{2}$, respectively. In Fig. 5 we again observed two absorption peaks in all the samples, one in the visible region due to the well-known SPR absorptions, which is 


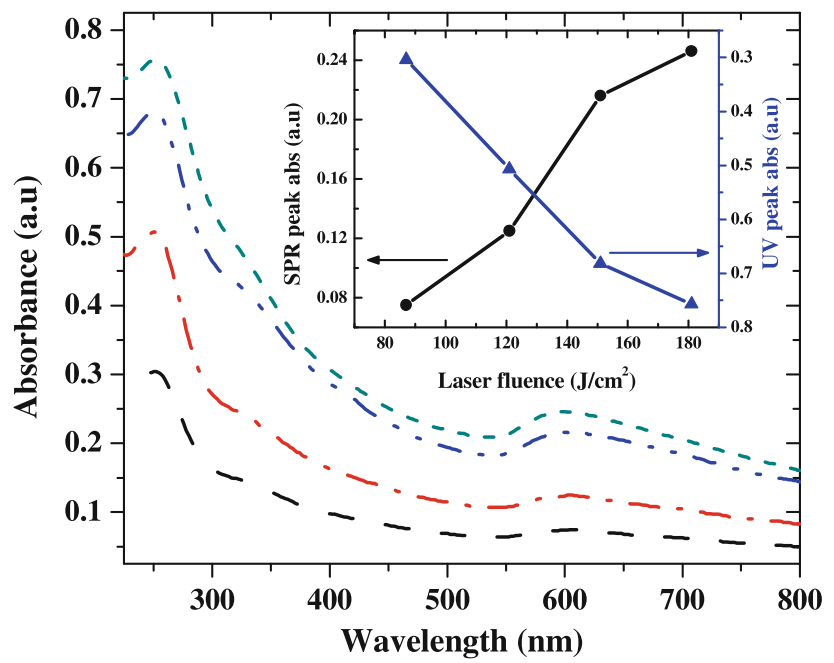

Fig. 5 Absorption spectra of $\mathrm{Cu}$ nanocolloids prepared in water medium with different laser fluence values. The wavelength of the used laser radiation is $1,064 \mathrm{~nm}$ and the laser ablation time duration is fixed at $20 \mathrm{~min}$. The colours black, red, blue and dark cyan correspond to laser fluence of $87,121,151$ and $181 \mathrm{~J} / \mathrm{cm}^{2}$, respectively. The inset shows the variations of the SPR peak absorbance and UV peak absorbance with the laser fluence

the characteristic absorption of metals and another in the UV region due to interband transition. The characteristics of both the SPR and UV peaks are affected by the variation of LF value. It is found that with increase in LF the SPR peak position is shifted to the shorter wavelength. This result suggests a decrease of particle size of the prepared $\mathrm{Cu}$ nanocolloids with increase in LF. In fact higher LF leads to larger kinetic energy that increases collision among initially formed large nanoparticles in the condensation process which further reduces the size (Haider et al. 2011). From the inset of Fig. 5 it is seen that the maximum value of SPR peak as well as that of UV absorption peaks varies with the LF. It is found that UV absorption peak intensity increases with the increase of the LF continuously whereas the SPR absorption peak intensity increase slowly at first with the increase of LF and then it becomes constant. The reason for this is the same as has been explained earlier.

\section{Conclusions}

In conclusion, here we have reported the preparation of uncapped $\mathrm{Cu}$ nanoparticles having average sizes (radius) varying between 1.7 and $6 \mathrm{~nm}$ by laser ablation of solid copper target in water medium by using Nd:YAG laser radiation at $1,064 \mathrm{~nm}$ having $10 \mathrm{~ns}$ pulse duration and by varying the laser ablation time duration and laser fluence. The UV-visible absorption spectra of all the samples show the presence of absorption peaks in the visible (varying between 593 and $607 \mathrm{~nm}$ ) due to SPR and interband absorptions $(\sim 251 \mathrm{~nm})$ in Cu nanoparticles. The shift of the SPR peak wavelength had taken place due to the variation in the size of the prepared nanoparticles and the linewidth of the SPR peaks increased with the reduction in sizes of the prepared nanoparticles due to intrinsic size effects. Due to the absence of any capping agent or surfactant molecules and quantum size effect the sharp absorption in the interband region is observed. The measured experimental data of SPR linewidth are fitted with a theoretical expression in the framework of Mie theory and the estimated value of bulk linewidth $\left(\gamma_{0}\right)$ and the phenomenological parameter $\mathrm{A}$ are $89 \mathrm{meV}$ and 0.1 , respectively.

Acknowledgments The authors are grateful to DST, Grant No. SR/ FTP/PS-67/2008, Government of India, for the partial financial support. We are thankful to the reviewer for his/her valuable advices.

Open Access This article is distributed under the terms of the Creative Commons Attribution License which permits any use, distribution, and reproduction in any medium, provided the original author(s) and the source are credited.

\section{References}

Ahmadi TS, Wang ZL, Green TC, Henglein A, El-Sayed MA (1996) Shape controlled synthesis of colloidal platinum nanoparticles. Science 272:1924-1925

Alvarez MM, Khoury TJ, Schaaff GT, Shafigullin NM, Vezmar I, Whetten LR (1997) Optical absorption spectra of nanocrystal gold molecules. J Phys Chem B 101:3706-3712

Balamurugan B, Maruyama T (2005) Evidence of an enhanced interband absorption in $\mathrm{Au}$ nanoparticles: size dependent electronic structure and optical properties. Appl Phys Lett 87:30510-30513

Berciaud S, Cognet PT, Lounis B (2005) Observation of intrinsic size effects in the optical response of individual gold nanoparticles. Nanolett 5:515-518

Chen CQ, Shen Q, Gao H (2007) Formation of nanoparticles in waterin-oil Micro emulsions controlled by the yield of hydrated electron: the controlled reduction of $\mathrm{Cu}^{+2}$. J Colloid Interface Sci 308:491-499

Curtis A et al (1988) Preparation and structural characterization of an unprotected copper sol. J Phys Chem 92:2270-2275

Dolgaev SI, Simakin AV, Voronov VV, Shafeev GA, Verduraz FB (2002) Nanoparticles produced by laser ablation of solids in liquid environment. Appl Surf Sci 186:546-551

Frederix F, Friedt JM, Choi KH, Campitelli A, Mondelaers D, Maes G, Borghs G (2003) Biosensing based on light absorption of nanoscaled gold and silver particles. Anal Chem 75:6894-6900

Haider AFMY, Sengupta S, Abedin KM, Aminul I (2011) Fabrication of gold nanoparticles in water by laser ablation technique and their applications. Appl Phys A 11:6542-6550

Huang HH, Ni XP, Loy GL, Chew CH, Tan KL, Loh FC, Deng JF, Xu GQ (1996) Photochemical formation of silver nanoparticles in poly (N-Vinyl pyrrolidon). Langmuir 12:909-912

Jeon JS, Yeh C, Chin J (1998) Studies of silver nanoparticles by laser ablation method. Chem Soc 45:721-726

Kazakevich PV, Vorono VV, Simakin AV, Shafeev GA (2004) Production of copper and brass nanoparticles upon laser ablation in liquid. Quantum Electron 34:951-956 
Khaydarov RA et al (2009) Electrochemical method for the synthesis of silver Nanoparticles. Electrochem Commun 8:227-230

Kreibig U, Genzel U (1985) Optical absorption of small metallic particles. Surf Sci 156:678-700

Kreibig U, Vollmer M (1995) Optical properties of metal clusters. Springer-Verlag, Berlin

Lee KS, El-Sayed MA (2006) Gold and Silver nanoparticles in sensing and imaging: Sensitivity of plasmon response to size, shape and metal composition. J Phys Chem B 110:19220-19225

Lee J, Kim DK, Kang W (2006) Preparation of Cu nanoparticles from copper powder dispersed in 2 propanol by laser ablation. Bull Korean Chem Soc 27:1869-1872

Link S, El-Sayed MA (1999) Spectral properties and relaxation dynamics of surface plasmon electronic oscillation in gold and silver nanodots and nanorods. J Phys Chem B 103:8410-8426

Link S, El-Sayed MA (1998) Size and temperature dependence of plasma absorption of colloidal gold nanoparticles. J Phys Chem B 103:4212-4217

Link S, Burda C, Nikoobakht B, El-Sayed MA (2000) Laser induced shape changes of colloidal gold nanorods using femtosecond and nanosecond laser pulses. J Phys Chem B 104:6152-6163

Mafune F, Kohno J, Takeda Y, Kondow T (2000) Formation and size control of silver nanoparticles by laser ablation in aqueous solution. J Phys Chem B 104:9111-9117

Mafune F, Kohno J, Takeda Y, Kondow T (2002) Full physical preparation of size selected gold nanoparticles in solution: Laser ablation and laser induced size control. J Phys Chem B 106:7575-7577

Nedersen J, Chumanov G, Cotton TM (1993) Laser ablation of metals: a new method for preparing SERS active nanocolloids. Appl Spectrosc 47:1959-1961

Pinchuk A, Plessen GV, Kreibig U (2004) Influence of interband electronic transition on the optical absorption in metallic nanoparticles. J Phys D 37:3133-3139

Prochazska M et al (1997) Probing application of laser ablated Ag colloids in SERS spectroscopy: improvement of ablation procédure and SEERS spectral testing. Anal Chem 69:5103-5106

Rao CNR, Kulkarni GU, Thomas PJ, Edwardy PP (2002) Size dependent chemistry: properties of nanocrystals. Chem A Euro J 8:28-35

Singh SC, Swarnakar RK, Gopal R (2010) Zn/ZnO core/shell nanoparticles synthesized by laser ablation in aqueous environment: optical and structural characterizations. Bull Mater Sci 33:21-26

Takami A, Kurita H, Koda S (1999) Laser induced size reduction of noble metal particles. J Phys Chem B 103:1226-1232

Yeh MS et al (1999) Formation and characteristics of $\mathrm{Cu}$ colloids from $\mathrm{CuO}$ powder by laser irradiation in 2-propanol. J Phys Chem 103:6851-6857

Yeshchenko OA, Dmitruk IM, Dmitruk AM, Alexeenko AA (2006) Influence of annealing conditions on structure and optical properties of copper nanoparticles embedded in silica matrix. Mater Sci Eng B 137:247-254 